REVISTA DE INVESTIGACIÓN EDUCATIVA DE LA REDIECH N. 8

\title{
Organización y financiamento de escuelas primarias públicas, en tres municipios de Chihuahua
}

\author{
David Manuel Arzola Franco \\ Claudia Celina Gaytán Díaz \\ Daniel Alarcón Nakamura \\ Profesores investigadores \\ Centro de Investigación y Docencia
}

\section{Resumen}

Este trabajo da cuenta de los resultados de un estudio que tiene el propósito de caracterizar los servicios de educación primaria en tres municipios del estado de Chihuahua: Ahumada, Chihuahua y Juárez. La investigación se realizó a partir de una encuesta cuyos instrumentos se aplicaron en 150 escuelas primarias públicas, seleccionadas de manera aleatoria, pertenecientes a los subsistemas estatal y federalizado. La muestra abarcó a 1,112 docentes, 169 directores, 106 auxiliares técnico pedagógicos (ATP) y 69 supervisores escolares.

Con respecto a la organización podemos decir que la mayoría de las escuelas se encuentran ubicadas en medios urbanos, donde la mayoría de los profesores laboran en el turno matutino y solo un $9 \%$ de ellos laboran en escuelas multigrado.
Se encontró que todavía hay un porcentaje muy elevado de profesores que atienden más de 30 estudiantes por grupo (37\%).

Hay una gran variedad de programas operando en las escuelas, aunque se destacan tres, Escuela segura (69.8\%), el Programa Nacional de Lectura (33.1\%) y Escuelas de calidad (31.4\%).

En lo referente al financiamiento se observa que a pesar de ser escuelas públicas deben buscar de manera permanente los medios para sufragar los gastos que las actividades cotidianas demandan. Además de los recursos que aporta el Estado la participación de los padres de familia representa un apoyo indispensable. Las aportaciones de particulares o asociaciones civiles son prácticamente inexistentes.

Palabras clave: organización escolar, centros educativos, financiamiento. 


\section{Introducción}

El presente trabajo se deriva de un proyecto de investigación que se lleva a cabo en el Centro de Investigación y Docencia, institución de posgrado perteneciente a los Servicios Educativos del Estado de Chihuahua, quién ha desarrollado el proyecto en coordinación con el Centro Chihuahuense de Estudios de Posgrado.

Durante la última década la educación básica se encuentra sometida a un proceso continuo de propuestas reformadoras, en el caso de la educación primaria en 2009 se integra al proceso de Reforma Integral de la Educación Básica, cuya plataforma estratégica planteó la incorporación de un nuevo plan de estudios en el que se enuncian cuatro campos formativos: lenguaje y comunicación; pensamiento matemático; desarrollo personal y para la convivencia; y exploración y comprensión del mundo natural y social (SEP, 2009). No obstante, para 2011 , con la idea de articular los tres niveles educativos se expide el Acuerdo 592 en el que se hacen algunos planteamientos adicionales al proceso reformador que estaba ya en marcha en la educación primaria.

Este escenario ha planteado desafíos importantes a los docentes y los directivos quienes deben enfrentar el reto de poner en marcha un currículo nacional al tiempo que

...la investigación y la experiencia recogida en los sistemas educativos indican que las innovaciones son inseparables de los contextos y procesos institucionales entre los que deben encontrar su lugar y que, por ello, su construcción adquiere un carácter político. Tan político como las orientaciones y decisiones de gobierno que les dan origen y como el tipo de estructuras que su realización compromete; entre ellas, las formas de la administración que sustenta la gestión de las escuelas, así como el gobierno y organización de éstas, que forman parte de su cultura, sus prácticas y su hacer cotidiano (Ezpeleta, 2004: 406).

Considerando estos elementos como punto de partida, el interés se centra en el rescate de las opiniones de los sujetos motivo de estudio: docentes, directivos y asesores técnico-pedagógicos (ATP), en relación con un conjunto de elementos que nos permitan caracterizar la estructura organizativa de los centros escolares (Ara, Bonals, González, Riu, y Sardans, 2005), tales como el tipo de organización, el número de alumnos por grupo, las actividades de apoyo o complementarias, los programas y proyectos que se operan al interior de las escuelas y los medios para el financiamiento.

\section{Estrategia metodológica}

El estudio se realizó a partir de la aplicación de una encuesta a una muestra representativa de sujetos, de las escuelas primarias públicas, estatales y federalizadas, de tres municipios del Estado de Chihuahua.

El equipo de investigadores, con la colaboración de los alumnos de tres grupos de maestría, elaboró los ítems para el estudio tipo encuesta. A partir de este trabajo se diseñaron los instrumentos para docentes, directores y estudiantes.

Los instrumentos se sometieron a una prueba piloto, en la que se realizaron dos aplicaciones con una diferencia de dos se- 
manas entre la primera y la segunda aplicación. Posteriormente cada uno de los ítems se evaluó mediante un coeficiente de correlación para conocer el grado de estabilidad de los instrumentos. Los estadísticos de prueba utilizados fueron: " $\mathrm{r}$ " de Pearson para los ítems de opción múltiple, y Alpha de Cronbach para las escalas. A partir de esta evaluación se eliminaron los ítem que presentaban bajos puntajes de correlación, de tal manera que la versión final de los instrumentos presenta los siguientes índices de correlación: $\mathrm{r}=0.81$ y Alpha de Cronbach $=0.78$

Finalmente los cuestionarios se aplicaron en un total de 150 escuelas primarias, seleccionadas mediante un procedimiento aleatorio, la muestra comprende a 1,112 docentes, 169 directores, 106 ATP y 69 supervisores escolares.

\section{Análisis de resultados}

\section{El aspecto organizativo de las escuelas primaria}

El estado de Chihuahua tiene un sistema de educación básica escindido en dos subsistemas: el estatal y el federalizado. Uno es coordinado por la Secretaría de Educación, Cultura y Deporte del Estado, al que coti- dianamente se le denomina estatal y otro por los Servicios Educativos del Estado de Chihuahua (SEECH) a estos planteles y su personal se les conoce como federalizados. De los 150 centros escolares 78 pertenecen al subsistema federalizado lo que representa el 52\% de las escuelas consideradas en la muestra de escuelas primarias de los municipios de Juárez, Ahumada y Chihuahua; el $48 \%$ restante son escuelas estatales.

La mayoría de las escuelas (82\%) se encuentran ubicadas en medios urbanos debido a que el estudio fue efectuado en los dos centros poblacionales más grandes de la entidad: Cd. Juárez y Chihuahua, un 10\% en zonas semiurbanas y solo el $8 \%$ en el medio rural.

El $72 \%$ de los profesores encuestados trabajan en el turno matutino, los docentes del subsistema estatal, laboran de manera predominante por la mañana (81\%), en cambio el porcentaje de docentes federalizados para este turno es mucho menor (65\%). Por lo tanto encontramos mayor diversidad en el subsistema federalizado: el $16 \%$ de los docentes laboran en el turno vespertino, un $7.8 \%$ en escuelas con jornada ampliada, y un 10\% en doble turno (ver tabla 1), este último dato revela que hay un

\begin{tabular}{|c|c|c|c|c|c|c|c|}
\hline \multirow{2}{*}{\multicolumn{2}{|c|}{ Subsistema }} & \multicolumn{5}{|c|}{$\begin{array}{c}\text { Tabla } 1 \\
\text { Docentes por turno }\end{array}$} & \multirow[b]{2}{*}{ Total } \\
\hline & & \multirow{2}{*}{\begin{tabular}{|r|} 
Matutino \\
425
\end{tabular}} & \multirow{2}{*}{$\begin{array}{r}\text { Vespertino } \\
60\end{array}$} & \multirow{2}{*}{\begin{tabular}{|r|}
$\begin{array}{c}\text { Jornada } \\
\text { ampliada }\end{array}$ \\
13
\end{tabular}} & \multirow{2}{*}{\begin{tabular}{|r|}
$\begin{array}{l}\text { Doble } \\
\text { turno }\end{array}$ \\
25
\end{tabular}} & \multirow{2}{*}{\begin{tabular}{|r|}
$\begin{array}{c}\text { No } \\
\text { contestó }\end{array}$ \\
2
\end{tabular}} & \\
\hline Estatal & Recuento & & & & & & 525 \\
\hline & $\%$ & $38.2 \%$ & $5.4 \%$ & $1.2 \%$ & $2.2 \%$ & $.2 \%$ & $47.2 \%$ \\
\hline \multirow[t]{2}{*}{ Federalizado } & Recuento & 378 & 92 & 45 & 58 & 3 & 576 \\
\hline & $\%$ & $34.0 \%$ & $8.3 \%$ & $4.0 \%$ & $5.2 \%$ & $.3 \%$ & $51.8 \%$ \\
\hline \multirow[t]{2}{*}{ No contestó } & Recuento & 5 & 0 & 0 & 3 & 3 & 11 \\
\hline & $\%$ & $.4 \%$ & $.0 \%$ & $.0 \%$ & $.3 \%$ & $.3 \%$ & $1.0 \%$ \\
\hline \multirow[t]{2}{*}{ Total } & Recuento & 808 & 152 & 58 & 86 & 8 & 1112 \\
\hline & $\%$ & $72.7 \%$ & $13.7 \%$ & $5.2 \%$ & $7.7 \%$ & $.7 \%$ & $100.0 \%$ \\
\hline
\end{tabular}


porcentaje importante de maestros federalizados -uno de cada diez- que tienen doble plaza, mientras que solo el $4.8 \%$ de los estatales trabajan en doble turno.

El $91 \%$ de los profesores trabajan es escuelas de organización completa, es decir, cada grado escolar es atendido por un profesor, solo el 5\% de los encuestados trabajan en escuelas multigrado, esta situación es comprensible puesto que el estudio se efectuó en los dos municipios con mayor población urbana de la entidad: Chihuahua y Cd. Juárez: encontramos que en Ahumada prácticamente una cuarta parte de docentes (24.4\%) trabajan en escuelas multigrado, mientras que en Juárez se reporta solo un $5.2 \%$, y en Chihuahua apenas un .72\%

En cuanto al número de alumnos por grupo que se atienden (ver tabla 2), encontramos todavía un porcentaje muy elevado de profesores que tienen más de 30 estudiantes (37\%), prácticamente cuatro de cada 10 docentes. Y este problema es toda- vía más agudo en el caso de los federalizados ya que casi la mitad de ellos (47.8\%) tienen más de 30 alumnos en su grupo, en contraste con el $25.5 \%$ de profesores del subsistema estatal que se encuentran en esa circunstancia.

Aunque el 89.6\% de los profesores encuestados están frente a grupo, se encontró un $10 \%$ que realizan actividades que hemos denominado "de apoyo o complementarias" entre los que destacan los de educación física 3.5\%, de USAER 2.5\% y de actividades culturales o artísticas $2.4 \%$. En la distribución por subsistema podemos observar que no hay mucha diferencia con respecto a las cifras generales: profesores de educación física $4.4 \%$ en el subsistema estatal y $2.8 \%$ en el federalizado, de USAER $2.3 \%$ estatales y $2.6 \%$ federalizados, y de actividades culturales o artísticas $2.7 \%$ estatales y $2.1 \%$ federalizados. Estos datos evidentemente señalan un déficit de profesores de apoyo a las actividades educativas complementarias (ver tabla 3).

\begin{tabular}{|c|c|c|c|c|c|c|c|c|c|}
\hline \multirow{2}{*}{\multicolumn{2}{|c|}{ Subsistema }} & \multicolumn{7}{|c|}{$\begin{array}{c}\text { Tabla } 2 \\
\text { Alumnos por grupo }\end{array}$} & \multirow{2}{*}{ Total } \\
\hline & & $\begin{array}{c}\text { Menos } \\
\text { de } 21\end{array}$ & $\begin{array}{l}\text { De } 21 \\
\text { a } 30\end{array}$ & $\begin{array}{c}\text { De } 31 \\
\text { a } 40\end{array}$ & $\begin{array}{c}\text { De } 41 \\
\text { a } 50\end{array}$ & \begin{tabular}{|c|} 
De $51 \mathrm{a}$ \\
60
\end{tabular} & $\begin{array}{l}610 \\
\text { más }\end{array}$ & $\begin{array}{c}\text { No } \\
\text { contestó }\end{array}$ & \\
\hline \multirow[t]{2}{*}{ Estatal } & Recuento & 61 & 319 & 121 & 1 & 1 & 11 & 11 & 525 \\
\hline & $\%$ & $5.5 \%$ & $28.7 \%$ & $10.9 \%$ & $.1 \%$ & $.1 \%$ & $1.0 \%$ & $1.0 \%$ & $47.2 \%$ \\
\hline \multirow[t]{2}{*}{ Federalizado } & Recuento & 69 & 226 & 248 & 18 & 0 & 9 & 6 & 576 \\
\hline & $\%$ & $6.2 \%$ & $20.3 \%$ & $22.3 \%$ & $1.6 \%$ & $.0 \%$ & $.8 \%$ & $.5 \%$ & $51.8 \%$ \\
\hline \multirow[t]{2}{*}{ No contestó } & Recuento & 3 & 5 & 2 & 0 & 0 & 0 & 1 & 11 \\
\hline & $\%$ & $.3 \%$ & $.4 \%$ & $.2 \%$ & $.0 \%$ & $.0 \%$ & $.0 \%$ & $.1 \%$ & $1.0 \%$ \\
\hline \multirow[t]{2}{*}{ Total } & Recuento & 133 & 550 & 371 & 19 & 1 & 20 & 18 & 1112 \\
\hline & $\%$ & $12.0 \%$ & $49.5 \%$ & $33.4 \%$ & $1.7 \%$ & $.1 \%$ & $1.8 \%$ & $1.6 \%$ & $100.0 \%$ \\
\hline
\end{tabular}


REVISTA DE INVESTIGACIÓN EDUCATIVA DE LA REDIECH N. 8

Tabla 3

Docentes de actividades de apoyo o complementarias

\begin{tabular}{|l|l|l|l|l|l|l|l|l|l|}
\hline & $\begin{array}{l}\text { Docente } \\
\text { frente a } \\
\text { grupo }\end{array}$ & $\begin{array}{l}\text { Actividades } \\
\text { culturales y } \\
\text { artísticas }\end{array}$ & $\begin{array}{l}\text { Apoyo } \\
\text { USAER }\end{array}$ & $\begin{array}{l}\text { Computa- } \\
\text { ción }\end{array}$ & $\begin{array}{l}\text { Educación } \\
\text { física }\end{array}$ & $\begin{array}{l}\text { Lengua } \\
\text { adicional }\end{array}$ & Otra & $\begin{array}{l}\text { No con- } \\
\text { testó }\end{array}$ & $\begin{array}{l}\text { Total } \\
\text { Recuento }\end{array}$ \\
996 & 27 & 28 & 4 & 39 & 8 & 7 & 3 & 1112 \\
\hline$\%$ & $89.6 \%$ & $2.4 \%$ & $2.5 \%$ & $.4 \%$ & $3.5 \%$ & $.7 \%$ & $.6 \%$ & $.3 \%$ & $100.0 \%$ \\
\hline
\end{tabular}

En lo que concierne a los programas establecidos formalmente en estas escuelas, se destacan tres, los datos se toman del cuestionario de directivos sin embargo las cifras presentadas por los docentes tienen un comportamiento similar (ver Figura 1): Escuela segura (69.8\%), Programa Nacional de Lectura (33.1\%) y Escuelas de calidad (31.4\%): Con porcentajes más bajos: Programa escuela y salud (20.7\%), Educación preventiva contra el consumo de drogas o DARE (14.8\%), Escuela de tiempo completo (10.7\%) Red escolar (6.5\%) y Red de acciones educativas a favor de la equidad (1.8\%). Aunque con proporciones diferentes los ATP y supervisores ubican los programas en forma similar (ver Figura 2).
Estas cifras nos hablan de la manera en que el contexto interno y las condiciones externas van configurando las prioridades para asumir la responsabilidad de sacar adelante un programa: los municipios donde se encuentran las escuelas estudiadas han vivido episodios de violencia relacionada con el crimen organizado, de ahí que programas como Escuela Segura se trabajen en más del $60 \%$ de los centros educativos. Por otra parte, el impulso a la lectura ha sido marcado en los últimos años como una de las prioridades de la educación básica, y Escuelas de Calidad representa una fuente de ingreso económico para las instituciones por lo que también es un fuerte incentivo para que los colectivos se com-

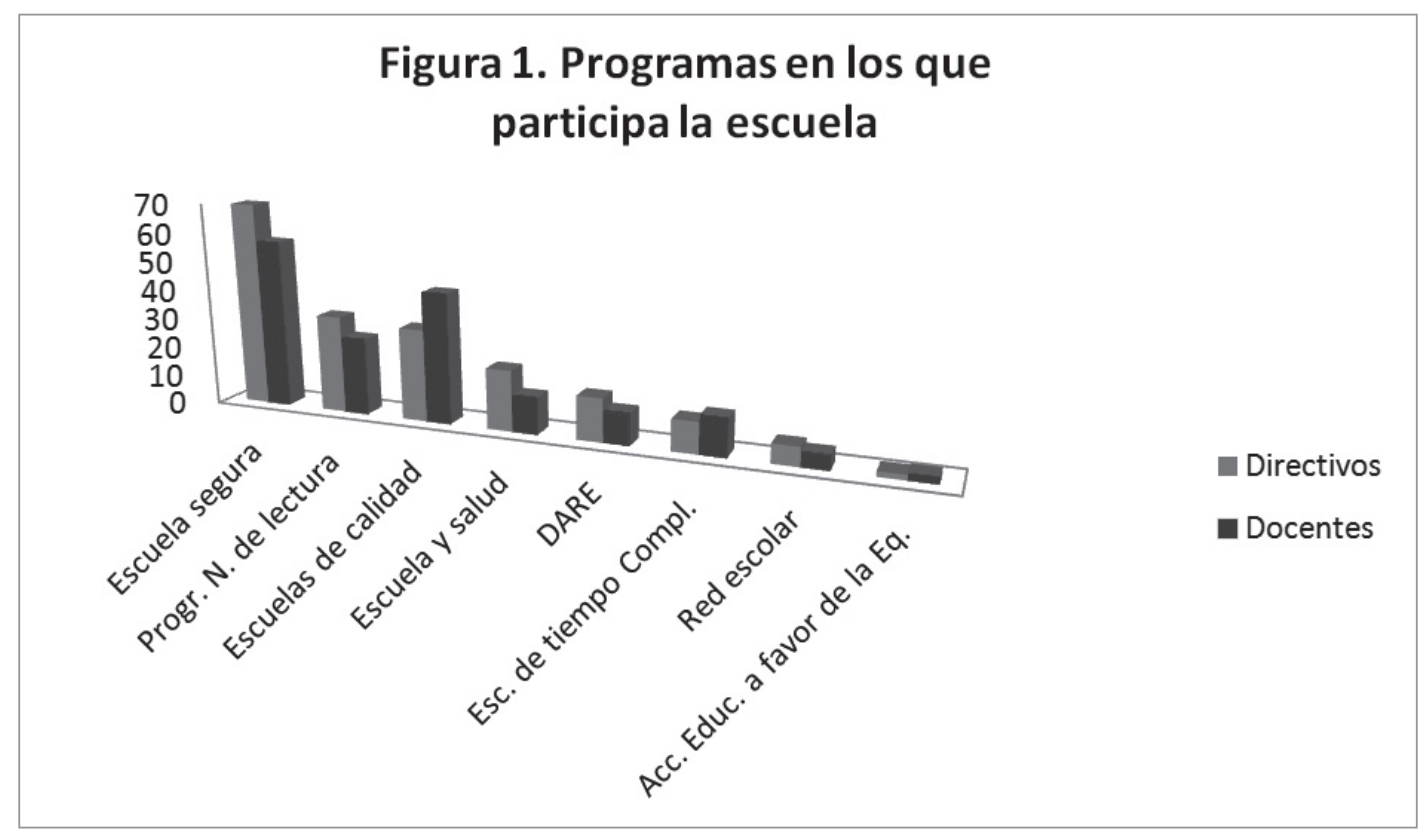




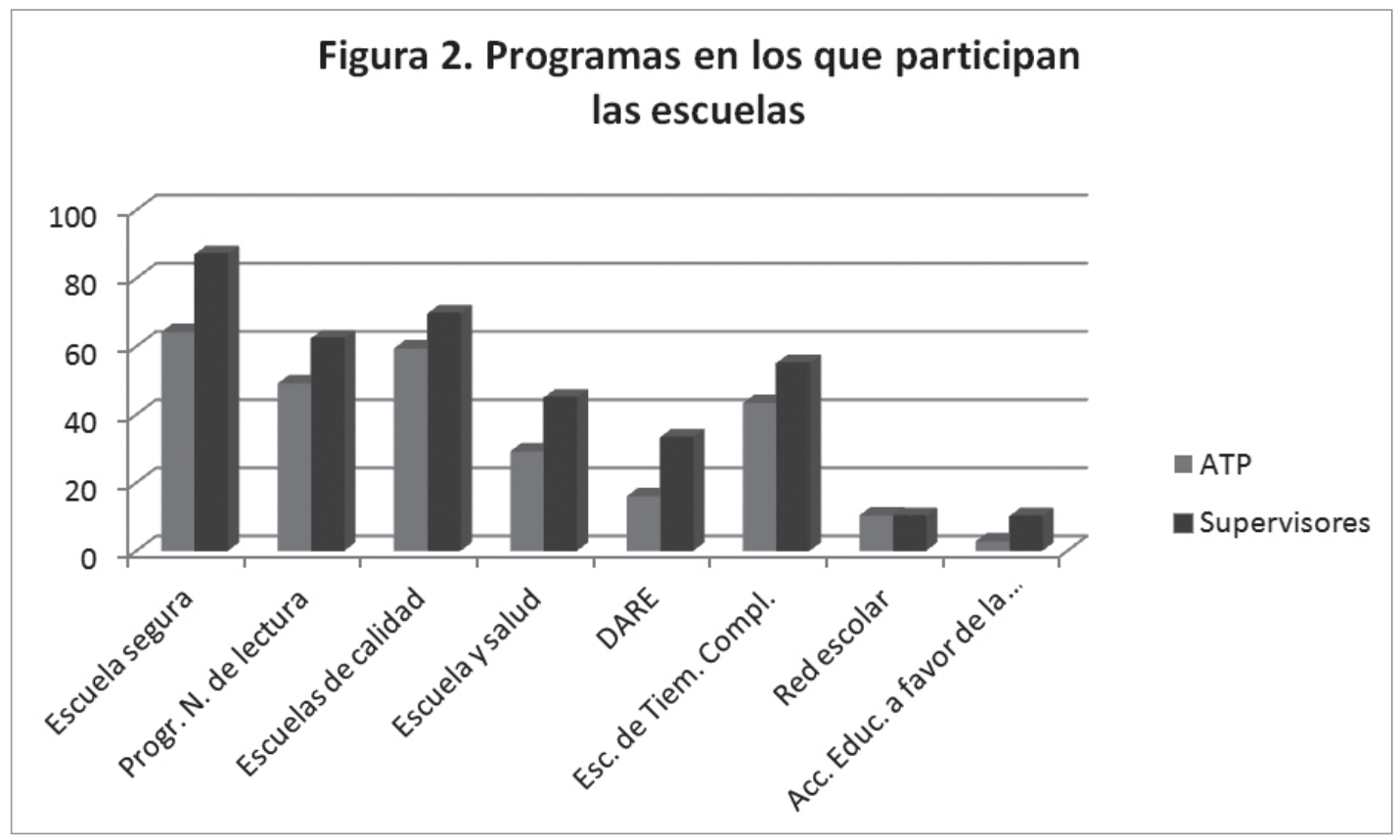

prometan en esta tarea, de hecho cuando se preguntó a los docentes y directivos sobre el principal beneficio que obtienen al participar en el Programa Escuelas de Calidad: el $47 \%$ de los docentes y el $41.2 \%$ de los directores, dicen que es para mejorar las condiciones materiales de los planteles, cifras que coinciden con la precepción de supervisores y jefes de sector: $49.2 \%$; como se puede observar, la obtención de recursos es uno de los detonadores más importantes para inscribirse en este programa, resulta

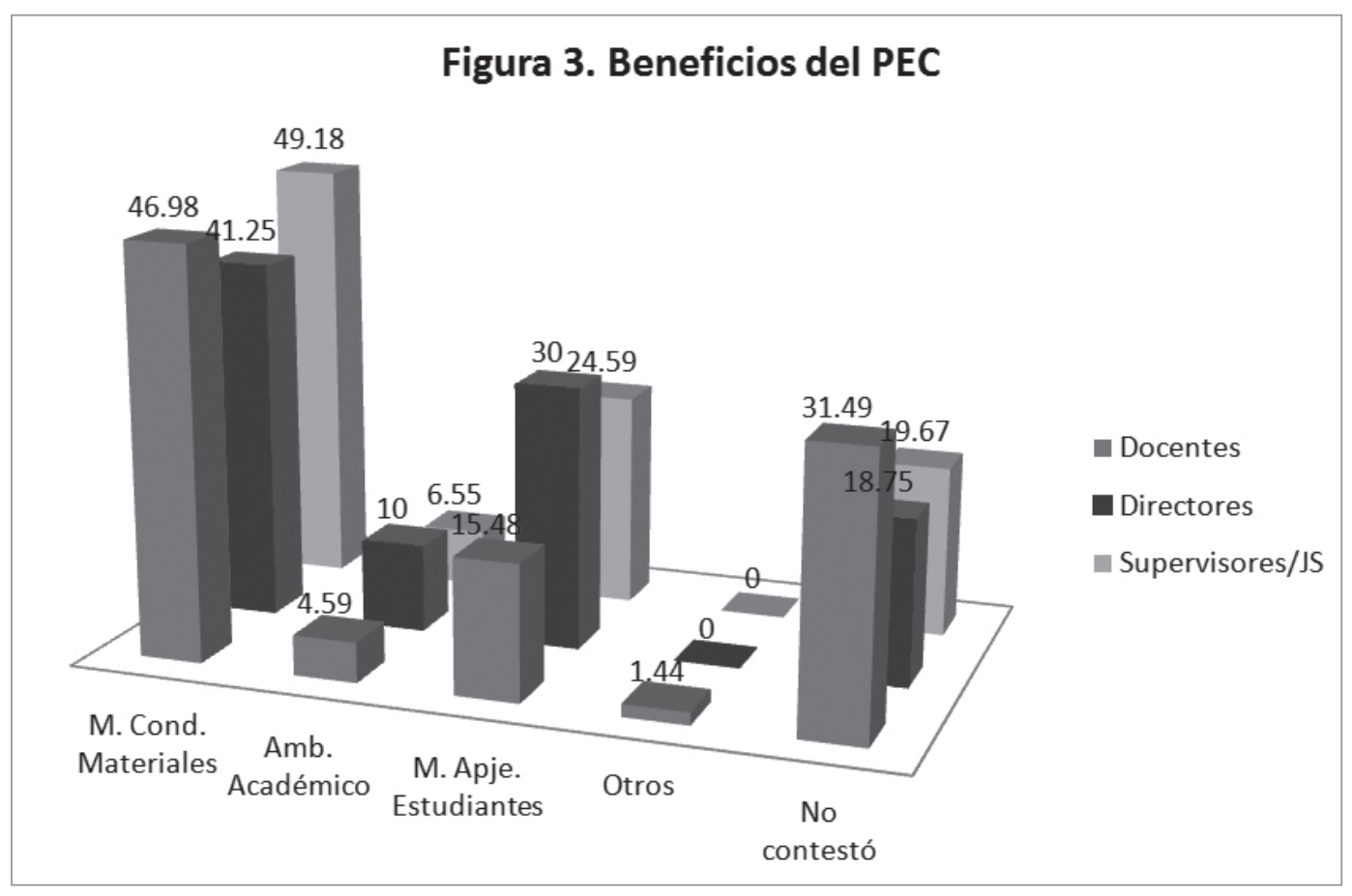

ABRIL—SEPTIEMBRE 2014 
además revelador que solamente el 15.5\% de los docentes, $30 \%$ de directores y $24.6 \%$ de supervisores y jefes de sector consideran como principal beneficio del PEC el mejorar el aprendizaje de los estudiantes (ver Figura 3), lo cual demuestra que el cambio educativo es una empresa difícil (Pozner, 2005), los movimientos inerciales se imponen a las tendencias innovadoras.

Con respecto a las escuelas con jornada ampliada, que corresponden al modelo propuesto a través del Programa Escuelas de Tiempo Completo (PETC), se puede observar que alcanzan un 16\% para el caso de los federalizados, mientras que se encontró solo un $9.7 \%$ en el caso de los docentes estatales. En conjunto, solamente el $12.9 \%$ de los docentes y 10.7 de los directores encuestados laboran en instituciones que trabajan con el PETC.

\section{II.El financiamiento}

Desde el punto de vista legal, las escuelas públicas están bajo el amparo del Estado para cubrir las necesidades que demanda la atención de los estudiantes, derechos consignados en el Artículo $3^{\circ}$ Constitucional, mas nunca ha sido un secreto que los recursos que se reciben por la parte oficial son insuficientes para el sostenimiento cotidiano de los planteles. De ahí que el personal de cada centro educativo tenga que hacer sus propias gestiones para sufragar los gastos que forzosamente deben enfrentar.

Los datos obtenidos confirman que las escuelas estudiadas, además de los recursos que reciben por parte de las instancias gubernamentales, tienen otras fuentes de financiamiento como son las actividades de recaudación que se organizan de manera interna, las cooperativas o tiendas escolares, la participación de los padres de familia y de manera escasa y aislada reciben donaciones o aportaciones de particulares $\mathrm{o}$ asociaciones civiles.

De acuerdo con las cifras obtenidas el mobiliario es el que obtuvo un mayor porcentaje de menciones con respecto al apoyo gubernamental, un $48.9 \%$ de los docentes y un $49.1 \%$ de los directores así lo señalaron. Mientras que solo un 14\% de los profesores y $37.9 \%$ de los directores dicen que el gobierno apoya con material de limpieza; con papelería y artículos de oficina: 7.6\% docentes y $21.9 \%$ directores; y con recursos para el mantenimiento de las escuelas: $3.8 \%$ docentes y $4.1 \%$ directores.

A los supervisores y jefes de sector también se les preguntó sobre el tipo de recursos con los que apoya principalmente el gobierno a las escuelas bajo su responsabilidad, se encontró que hay una brecha importante entre sus percepciones y las de docentes y directores, el $73.5 \%$ dicen que se apoya con mobiliario, $54.4 \%$ con material de limpieza, $51.5 \%$ con artículos de oficina. Mientras que cuando se trata de recursos para el mantenimiento de las escuelas, las cifras coinciden con lo apuntado por los otros sujetos: $7.4 \%$.

Cuando se les preguntó a los docentes sobre algún otro tipo de apoyos que se reciben por parte del Gobierno, el 1\% de los encuestados anotó lo siguiente: enciclomedias en el 2003, material deportivo, despensas DIF, despensas escolares, el pago de los maestros, infraestructura, el pago de luz y 


\begin{tabular}{|c|c|c|c|}
\hline Apoy & o gubernan & la escuela & \\
\hline Docent & & & \\
\hline Equipo & Recuento & 176 & 936 \\
\hline & $\%$ & $15.8 \%$ & $84.2 \%$ \\
\hline Mobiliario & Recuento & 544 & 568 \\
\hline & $\%$ & $48.9 \%$ & $51.1 \%$ \\
\hline Material & Recuento & 156 & 956 \\
\hline limpieza e higiene & $\%$ & $14.0 \%$ & $86.0 \%$ \\
\hline Papelería $\quad \mathrm{y}$ & Recuento & 84 & 1028 \\
\hline artículos de oficina & $\%$ & $7.6 \%$ & $92.4 \%$ \\
\hline Mantenimiento & Recuento & 42 & 1070 \\
\hline & $\%$ & $3.8 \%$ & $96.2 \%$ \\
\hline
\end{tabular}

agua, pintura para muros, útiles escolares, PEC.

Por otra parte, un $64.5 \%$ de los profesores afirman que tienen información sobre las actividades de recaudación de la escuela, y un $65.8 \%$ en relación con la cooperativa o tienda escolar y un $77.4 \%$ sobre las aportaciones de los padres de familia. Este último es uno de los temas más sensibles en la actualidad, aunque los datos nos confirman que se trata de una práctica que tiene un profundo arraigo en la cultura de las escuelas públicas de educación básica, siempre se ha manejado como una aportación "voluntaria" ya que desde el punto de vista legal el Estado tiene la obligación de brindar este servicio de manera gratuita.

Dentro del conjunto de actividades para recabar fondos en la escuela, las aportaciones de padres y madres de familia son la opción más socorrida, el 56\% de los directivos la consideran la primera opción para obtener dividendos, contra el $17 \%$ que señalan a la tienda o cooperativa escolar y el 16\% que optan por los festivales (ver Figura 4). Solamente el $1.2 \%$ de los directores dicen

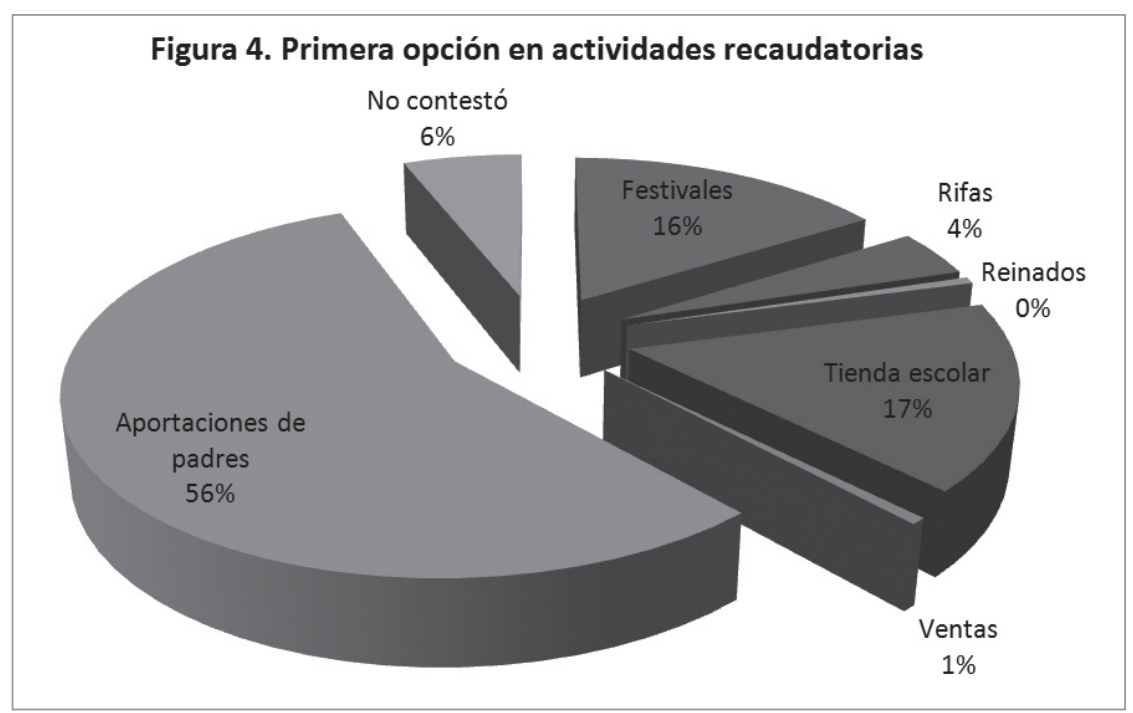


REVISTA DE INVESTIGACIÓN EDUCATIVA DE LA REDIECH N. 8

Figura 5. Aportación anual por padre/madre de familia

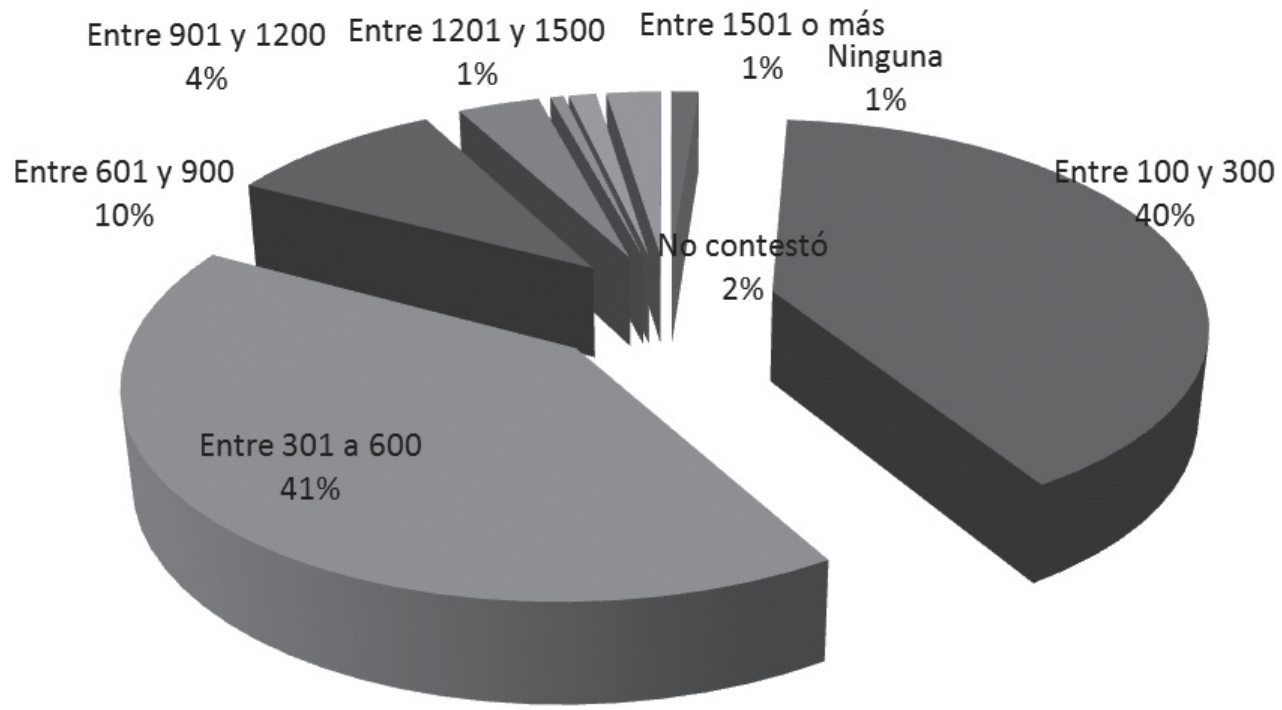

que no reciben ninguna aportación de los padres de familia; la mayoría, el $81.6 \%$, dicen que la cantidad que la escuela obtiene está entre 100 y 600 pesos al año por padre de familia; y el $17.3 \%$ indican que se reciben más de 601 pesos al año (ver Figura 5).

De igual manera se preguntó a los directivos sobre el porcentaje aproximado de padres/madres de familia que cumplen con las aportaciones, el 62.7\% dicen que el porcentaje de padres que aportan está por encima del $60 \%$, mientras que hay un $32 \%$ de directores que indicaron que el porcentaje de padres que aportan está por debajo del 40\% (ver Figura 6).

Con respecto al destino de esos recursos, el $68 \%$ de los directores señalan que se destinan al mantenimiento de los planteles

\section{Figura 6. Porcentaje de padres/madres que cumplen con las aportaciones}

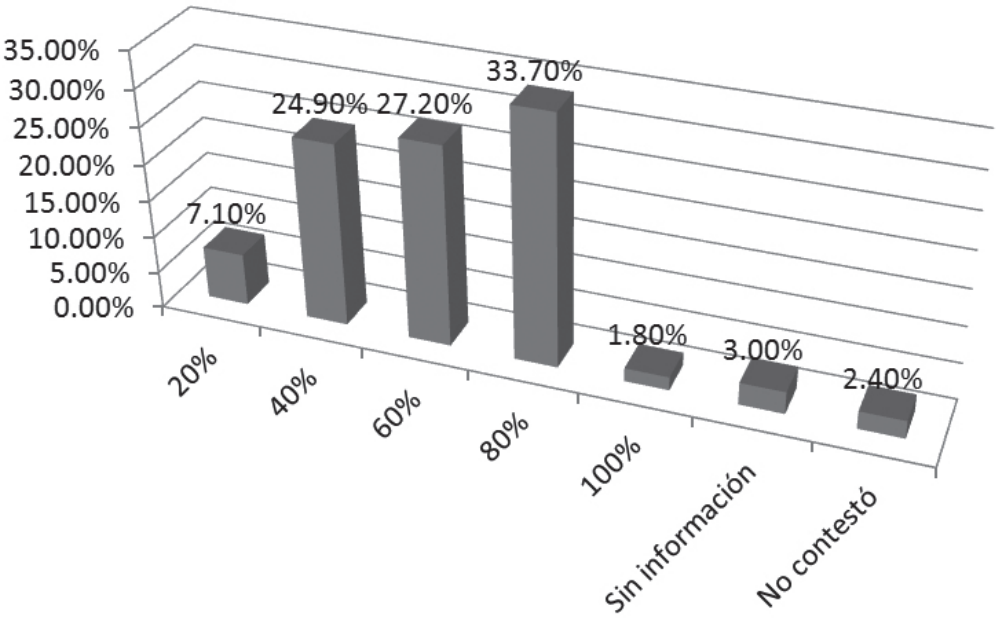




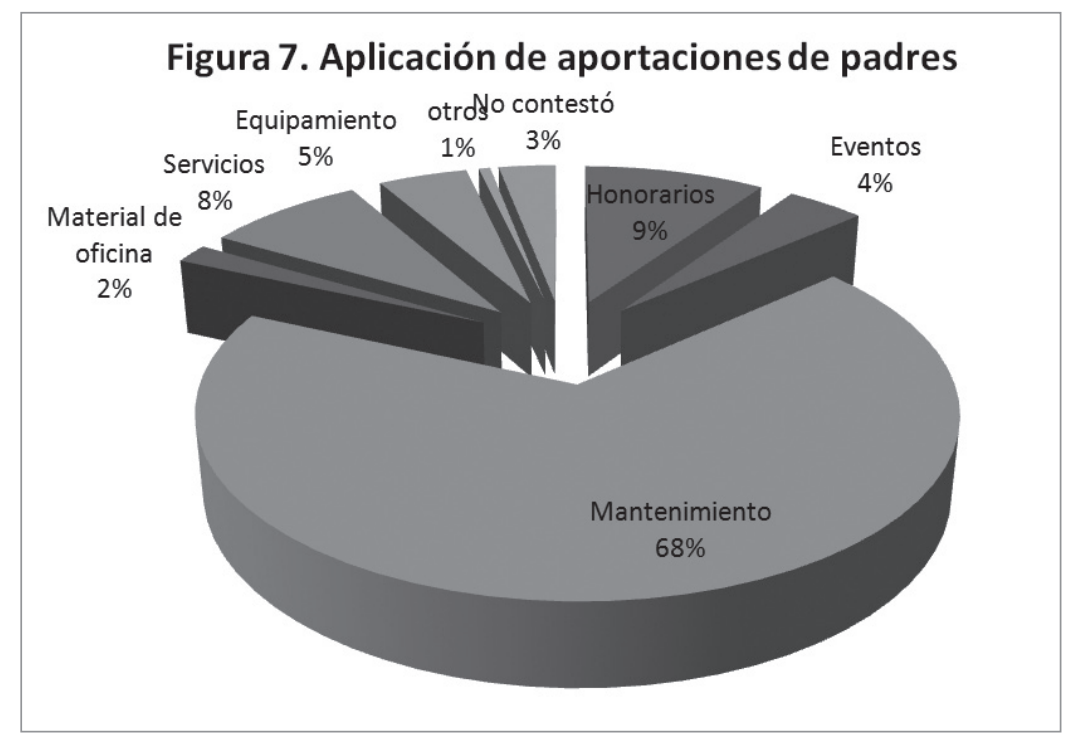

(ver Figura 7). A partir de estos datos se observa que la situación del financiamiento para las escuelas es preocupante entre muchas otras razones porque actualmente el titular de la SEP, con sus respectivas réplicas en las entidades federativas, ha prohibido este tipo de aportaciones. Es obvio que a pesar de esa disposición tanto directores como docentes siguen acudiendo a los padres para que apoyen económicamente a los planteles, pero la segunda preocupación tiene su origen precisamente en la arista de la equidad, puesto que en los medios socioeconómicos más desfavorecidos los estudiantes tienen también menos opciones para que les sean atendidas sus necesidades, tanto por la parte gubernamental como por la familiar.

Con respecto al apoyo de particulares o asociaciones civiles a las escuelas públicas, podemos constatar que la mayoría de los profesores dicen que no cuentan con este tipo de aportaciones: 9\% de los docentes y $14 \%$ de los directores dicen que se reciben apoyo en especie, un $2.5 \%$ de los docentes y $4.1 \%$ de los directores dicen que mate- riales para la construcción y solo un .6\% de docentes y $2.4 \%$ de directores dicen que reciben algún tipo de apoyo económico; las anotaciones que los docentes hacen respecto al tipo de apoyos que provienen de esta fuente son los siguientes: a veces jugos o cajitas de leche, apoyo psicológico para los alumnos, árboles del fondo unido, árboles y pintura, mobiliario.

\section{Conclusiones}

Con respecto a la organización podemos decir que la mayoría de las escuelas se encuentran ubicadas en medios urbanos debido a que el estudio fue efectuado en los dos centros poblacionales más grandes de la entidad. Asimismo la mayoría de los profesores laboran en el turno matutino (72\%), aunque con diferencias importantes por subsistema, los docentes del subsistema estatal, laboran de manera predominante por la mañana (81\%), en cambio el porcentaje de docentes federalizados para este turno es mucho menor (65\%). Se ubican en escuelas de organización completa el $91 \%$ de los profesores, aunque en Ahu- 
mada prácticamente una cuarta parte de docentes (24.4\%) trabajan en escuelas multigrado, mientras que en Juárez se reporta solo un 5.2\%, y en Chihuahua apenas un $.72 \%$ de docentes multigrado.

Un porcentaje muy elevado de profesores atienden más de 30 estudiantes por grupo (37\%), prácticamente cuatro de cada 10 docentes. Y este problema es todavía más agudo en el caso de los federalizados ya que casi la mitad de ellos (47.8\%) tienen más de 30 alumnos en su grupo, en contraste con el $25.5 \%$ de profesores del subsistema estatal que se encuentran en esa circunstancia.

La proporción de docentes que realizan actividades a las que hemos denominado "de apoyo o complementarias" es insignificante en relación al total de docentes que se ubican en las escuelas estudiadas, los profesores de educación física apenas llegan al 3.5\%, mientras que los profesores que se encargan de las USAER, actividades culturales o artísticas, lengua extranjera no rebasan el 3\% en cada caso.

Hay una gran variedad de programas operando en las escuelas, aunque no todos tienen el mismo nivel de penetración, es decir cada escuela trabaja con un cierto número de programas que se eligen de acuerdo a los criterios de cada centro educativo. Se destacan tres, Escuela segura (69.8\%), ligado básicamente a los problemas sociales relacionados con la violencia de los últimos años, el Programa Nacional de Lectura
(33.1\%) y Escuelas de calidad (31.4\%), en este último caso un número considerable de los sujetos encuestados declara que se adscriben a él con la finalidad de obtener recursos económicos.

En lo que se refiere al financiamiento se observa que a pesar de ser escuelas públicas deben buscar de manera permanente los medios para sufragar los gastos que las actividades cotidianas demandan. Además de los recursos que aporta el Estado la participación de los padres de familia representa un apoyo indispensable. Las aportaciones de particulares o asociaciones civiles son prácticamente inexistentes.

Los recursos que las escuelas obtienen por diversos medios, primordialmente las aportaciones de los padres se destinan básicamente al mantenimiento de los planteles.

\section{Referencias}

Ara, B., Bonals, J., González, A., Riu, M. A., y Sardans, A. (2005). Análisis de centros educativos. Barcelona: Horsori.

Ezpeleta, J. (2004). Innovaciones educativas. Reflexiones sobre los contextos en su implementación. En Revista Mexicana de Investigación Educativa. ABR-JUN, Vol. 9, Núm. 21, pp. 403-424.

Pozner, P. (2005). El directivo como gestor de aprendizajes escolares. Buenos Aires: Aique.

SEP (2009). Plan de estudios de educación primaria. Secretaría de Educación Pública. Consultado en: http://formacioncontinua.sep.gob.mx/sites/ReformaIntegral/RIEB_Plan_de_Estudios.php

SEP (2011). Acuerdo número 592 por el que se establece la articulación de la Educación Básica. México: SEP. 